\title{
CS11-04
}

\section{DISABILITY AND ICF CORE SETS IN MOOD DISORDERS}

J.L. Ayuso-Mateos ${ }^{1,2}$

${ }^{1}$ Department of Psychiatry, Universidad Autonoma de Madrid, ${ }^{2}$ Hospital Universitario de la Princesa, CIBERSAM, Madrid, Spain

The concept of disability has changed enormously, from a notion of handicap (ICIDM,1980) to the idea of person with disability $(I C F, 2001)$. The ICF considers three different levels of disability: body, person and environment, offering a possibility to address it in a universal, integrative and interactive perspective. The utility of the ICF in shifting the attention from a medical to a biopsychosocial perspective is therefore accepted. Having 1464 categories, it is hardly applicable to clinical practice and research. Mood disorders are characterized by a variety of psychiatric and somatic symptoms, associated with a significant loss of quality of life and functioning. Practical tools, such as Core Sets, that cover the spectrum of problems are needed. ICF Core Sets have been developed for depression and are currently being developed by our group for bipolar disorder. The ICF Comprehensive Core Sets for depression is the second larger among 12 Comprehensive ICF Core Sets for chronic disorders. This fact reflects the complex limitations in functioning and the numerous interactions with environmental factors. From the first version of the ICF Core Sets for depression as well as the preliminary studies for the bipolar disorder's core sets mental functions are mostly represented among the body functions domain. Few aspects important to mood disorders, as suicide, have been found to be relevant from both a systematic literature review and an expert survey in BD and in the consensus conference were the Core Sets for depression were establish but are not covered in the ICF. 